and consultation on euthanasia in the Netherlands' (SCEN) physicians. They usually serve as the official independent physician EAS consultants but they can also dispense less formal advice and assistance. The due care criteria set out in the Dutch EAS Act determine that the EAS physician is required to ensure that:

- the patient's request is voluntary and well considered

- the patient's suffering is unbearable and there is no prospect of improvement

- the patient has been informed about the situation and the prognosis

- there is no reasonable alternative in the patient's situation

- the patient has consulted at least one other, independent physician, who must see the patient and give a written opinion on whether the due care criteria set out above have been fulfilled

- due medical care and attention have been exercised in terminating the patient's life or assisting in suicide.

Kim and colleagues reviewed 66 psychiatric EAS cases from 2011 to 2014. The findings raise many questions. Seventy per cent of the patients were women and a quarter were 30-50 years old. Most patients had chronic, severe conditions and a history of attempted suicide; most were thought to have personality disorders and were described as socially isolated and lonely. There were also cases of psychosis, post-traumatic stress disorder, anxiety, somatoform disorders, eating disorders, as well as neurocognitive states, autism and prolonged grief. A quarter of the patients had EAS delivered by a physician new to them and although consultation with other physicians was extensive, in one-fifth of the cases there had been no independent psychiatric input, while in a quarter of the cases there was disagreement among consultants. In one case the euthanasia review committee found that it had failed to meet legal due care criteria.
The study findings raise many questions. How effectively are the criteria applied? How is capacity assessed? How can the physicians be so confident that there is no prospect for improvement in an individual's mental disorder? We still do not have clear and definitive indicators of outcome in these conditions. Most of the EAS patients had a diagnosis of depression, which inevitably would negatively influence their evaluations of their lives and future. Many people in the study felt lonely and isolated; in an affluent European country, couldn't there be psychosocial interventions to help improve people's quality of life? Most of these people were women. More questions can be raised but Pandora asks the fundamental question: is this really ethical?

Kim, S. Y. H., De Vries, R. G. \& Peteet, J. R. (2016) Euthanasia and assisted suicide of patients with psychiatric disorders in the Netherlands 2011 to 2014. JAMA Psychiatry (doi:10.1001/ jamapsychiatry.2015.2887)

\section{No health without mental health}

$\mathrm{M}$ ental health funding, both for research and service provision, has always lagged behind that for physical health and the global economic crisis, in recent years, has widened the gap. However, there is a glimpse of hope on the horizon. An initiative led by the World Health Organization may bring about a much needed change to the current state of affairs. A meeting is due to be held in April 2016, in Washington, DC, co-hosted by the World Bank Group and the World Health Organization. The meeting's aims are:

- to move mental health from the margins to the mainstream of the global health agenda by raising its profile

- to present the case for investing in mental health

- to identify entry points for renewed action and investment at the country, regional and global levels

- to mobilise a global coalition for action for scaled up implementation of mental health services for depression and anxiety in primary care and community settings.

\title{
Asylum Seekers and Refugees Mental Health Network
}

The Faculty of General Adult Psychiatry of the Royal College of Psychiatrists has launched seven networks, one of which is the Asylum Seekers and Refugees Mental Health Network. This network will allow interested psychiatrists to communicate with other colleagues in psychiatry through the sharing of information, knowledge and peer support. Its aims are:

- to allow psychiatrists working with asylum seekers and refugees to maintain an awareness of this changing and evolving area

- to provide a source of information on resources for this patient group

- to share information on the latest evidence base for treatment

- to offer peer support, allowing psychiatrists to share accounts of their clinical experience with this group.

The network leads are Dr Piyal Sen and Dr Katy Briffa. If you are interested in joining this network please contact the Faculty of General Adult Psychiatry Committee Manager, Lauren Wright, at lauren.wright@rcpsych.ac.uk. 